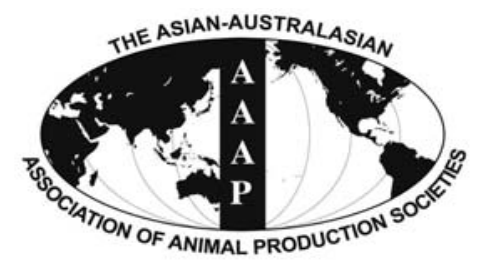

Asian-Aust. J. Anim. Sci.

Vol. 23, No. 2 : 162 - 168

February 2010

www.ajas.info

\title{
Eggshell Pigmentation Study in Blue-shelled and White-shelled Ducks
}

\author{
H. C. Liu, M. C. Hsiao, Y. H. Hu, S. R. Lee and W. T. K. Cheng ${ }^{1, *}$ \\ Ilan Branch, Livestock Research Institute, Council of Agriculture, Executive Yuan, Yilan 268, Taiwan
}

\begin{abstract}
This study attempted to clarify the difference in eggshell pigmentation between blue-shelled ducks (BSD) and whiteshelled ducks (WSD). The eggshell pigmentation deposition process is discussed. Ultraviolet spectro-photometer and HPLC were used to determine the biliverdin concentration in the shell gland, uterus liquid and eggshell at $6,12,18,20,23.5 \mathrm{~h}$ post-oviposition. The biliverdin concentration in the eggshell and uterus fluid showed significant differences between BSD and WSD, but not in the shell gland. The heme oxygenase activity in the shell gland of both kinds of ducks remained mostly constant during the ovulatory cycle with no variation. The assay of exogenous biliverdin injection into the shell gland antrum in the WSD indicated that exogenous biliverdin could be deposited continuously into the eggshell until the source was exhausted. A layer-by-layer dissolution assay was used to examine the eggshell pigment deposition process. The biliverdin concentration in the first to sixth layers of the eggshell in the BSD was significantly higher than that in the white-shelled counterpart. The blue pigment concentration increased persistently from the $6^{\text {th }}$ layer to the $1^{\text {st }}$ layer. The BSD eggshells did not accumulate a large quantity of biliverdin in the most external layer. They tended to increase the deposition layer by layer. Our results demonstrated that different BSD and WSD eggshell colors were influenced by the amount of biliverdin in the uterus fluid and not determined by the amount of biliverdin in the shell gland. This implies the existence of a mechanism that controls biliverdin transportation from the shell gland into the uterus fluid, thereby playing a key role in regulating duck eggshell color. (Key Words : Eggshell Pigmentation, Biliverdin, Blue-shelled Duck, Uterus Liquid)
\end{abstract}

\section{INTRODUCTION}

Avian eggshell pigmentation is a complex process involving two types of pigments, biliverdin and protoporphyrines. Biliverdin, a green pigment, is a byproduct of hemoglobin breakdown and will eventually display a blue or green color in the eggshell. Protoporphyrines, in contrast to biliverdin, is an immediate precursor of the heme molecule, and will give eggshells a reddish or brown color (Kennedy and Vevers 1975; Mikšík et al., 1994). The possible functions of egg coloration vary from those related to the physical properties of pigments (i.e., filtering solar radiation or strengthening the eggshell) to those related to the adaptive function of color. More recently, Moreno and Osorno (2003) proposed that blue and green egg colors may function as a post mating sexually selected signal of female phenotypic quality to their mates in order to induce a higher allocation of parental care.

\footnotetext{
* Corresponding Author: W. T. K. Cheng. Tel: +886-2-27337180, Fax: +886-2-27324070, E-mail: wtkcheng@ntu.edu.tw

1 Department of Animal Science and Technology, National Taiwan University, Taipei 106, Taiwan.

Received April 24, 2009; Accepted October 10, 2009
}

Gosler et al. (2005) proposed that the egg speckling caused by protoporphyrin pigments might compensate for reduced eggshell thickness due to calcium deficiency.

A previous study indicated that uterine tissues produce twice as much porphrin from delta-aminolevulenic acid compared to liver tissue in vitro (Polin, 1957). Later researches conducted experiments using brown eggshell hens $(\mathrm{BrSH})$ suggested that eggshell protoporphyrin is first synthesized in the shell gland, then secreted and deposited into the eggshells (Baird et al., 1975). The shell gland which secretes the cuticular layer is considered the final destination of pigment transportation (Solomon, 2002). Porphyrins are likely synthesized de novo in the cells where the process occurs. Biliverdin, on the other hand, is probably produced in erythrocytes (Kennedy and Vevers, 1973; Lang and Wells, 1987). Thus, these two principal eggshell pigments have very different origins, despite their molecular structure similarities. More recently, the biliverdin concentration in blue eggshell hens (BSH) and $\mathrm{BrSH}$ showed a significant difference in the shell gland with no differences in serum and bile. This demonstrates that the circulation system is not the biosynthesis site for shell-origin biliverdin. Biliverdin is most likely synthesized 
in the shell gland and deposited into the eggshell in chickens (Zhao et al., 2006).

The native Brown Tsaiya duck (Anas platyrhynchos) is a major laying duck breed in Taiwan. It is one of the highest laying duck breeds in the world (Tai et al., 1989; Chen et al., 2003). The eggshell colors of Brown Tsaiya vary from white to pale blue to dark blue. The eggshell color of the Pekin duck (Anas platyrhynchos) only white. Blue eggshells contain mainly biliverdin and a small amount of protoporphyrin. White eggshells contain only protoporphyrin (Liu et al., 1998). The genes involved in color polymorphism must exert their influence via enzyme systems that produce the different pigments in the eggshell. Heme oxygenation is the rate-limiting step for heme degradation, which produces biliverdin (Schwartz et al., 1980). The BSD shell gland may accumulate a higher biliverdin concentration than $\mathrm{BSH}$. The higher bioactivity of heme oxygenase in the BSD shell gland may be responsible for producing the higher biliverdin concentration. We compared the shell gland biliverdin concentrations and heme oxygenase activity between white eggshell ducks (WSD) and blue eggshell ducks (BSD) to clarify the differences in eggshell pigmentation. We also studied the eggshell pigmentation deposition process to elucidate the eggshell pigment deposition mechanism in ducks.

\section{MATERIALS AND METHODS}

\section{Animals and sample collection}

A total of 60 ducks including 30 laying Brown Tsaiya ducks with blue-shelled eggs and 30 Pekin ducks with white-shelled eggs were used in this study. Ducks were caged individually and fed ad libitum on a commercial layer diet as recommended by the NRC (1989). They were subjected to a cycle of $14 \mathrm{~h}$ light: $10 \mathrm{~h}$ darkness. When ducks reached 72 wks of age, the oviposition was monitored every $10 \mathrm{~min}$ during the night preceding sampling. Six Brown Tsaiya ducks and 6 Pekin ducks were selected randomly and sacrificed by electrical shock at 6,12 , 18,20 and $23.5 \mathrm{~h}$ post oviposition. After sacrifice, the shell gland was carefully cut to collect the uterine fluid for biliverdin concentration analysis. The shell gland and the eggs in the shell gland were then stored individually. Each shell gland pouch was divided into 2 parts for biliverdin concentration assay and heme oxygenase activity measurement, respectively. All samples were protected from light and stored at $-20^{\circ} \mathrm{C}$ until needed.

To study the eggshell pigment deposition process, five eggs each from BSD, WSD and WSD received a single dose biliverdin injection were collected from Brown Tsaiya and Pekin ducks, respectively.

\section{Analysis of biliverdin contents}

The shell gland was ground using liquid nitrogen into powder and weighted. The eggshell powder was then mixed with diluent $(50 \% 3 \mathrm{~N} \mathrm{HCl}$ and $50 \%$ acetonitrile) (HPLC grade, J. T. Baker, USA). The collected uterine liquid was dried using a speed vacuum and resuspended with diluent. All samples were centrifuged at $18,350 \times \mathrm{g}$ for $10 \mathrm{~min}$. The supernatants were analyzed using an HPLC method modified from Taylor et al. (2000). An L-4500 diode array detector, L-6200A intelligent pump, D-6000 interface (Hitachi, Japan) and Hamilton PRP-1 reversed phase (10 $\mu \mathrm{m}$ particle size, $4.1 \mathrm{~mm} \times 250 \mathrm{~mm}$ ) column were used. The flow rate was $2.0 \mathrm{ml} / \mathrm{min}$ and a solvent gradient was applied. The initial mobile phase composition was $20 \%$ acetonitrile and $80 \% \quad 0.1 \mathrm{M}$ ammonium acetate ( $\mathrm{pH}$ 5.2) (Sigma, Germany) for $2 \mathrm{~min}$. The solvent gradient consisted in a 16 min linear change to $100 \%$ acetonitrile, followed by $2 \mathrm{~min}$ at $100 \%$ acetonitrile. The total runtime was $20 \mathrm{~min}$. Calibrations were prepared as samples. Concentrations in the 6 calibration points were 31.25, 62.5, 125, 250, 500 and 2,000 nM with R values of 0.9984

The eggshells were rinsed with Milli-Q water (Millipore, MA) first and then dissolved without shell membranes in the mixed solution (methanol contained $5 \% \mathrm{H}_{2} \mathrm{SO}_{4}$ ) (HPLC grade, Riedel-deHaën, Germany) in the dark for $2 \mathrm{~d}$ at room temperature. The colored extract was filtered and subjected to spectrophotometer analysis. The UV spectro-photometric (Unicam, UK) method was used for the biliverdin in eggshell assay, performed at $670 \mathrm{~nm}$ to avoid impurity disturbance in the prepared samples. The sample quantification was performed using calibration curves constructed with standard solutions. Calibrations were prepared as samples. The concentrations in the 5 calibration points were $8,4,2,1$, and $0.5 \mu \mathrm{M}$ with $\mathrm{R}$ values of 0.9998 in UV spectrophotometry.

\section{Heme oxygenase activity assay}

The heme oxygenase activity assay followed the modified procedures of Ryter et al. (2000). A shell gland, 1 $\mathrm{cm}^{3}$ in size, was transferred into the eppendorf and homogenized well using scissors. One ml solution A $(0.25$ $\mathrm{M}$ sucrose, $20 \mathrm{mM}$ Tris- $\mathrm{HCl}, \mathrm{pH}$ 7.4) and $1 \mu$ protease inhibitor cocktail were then added. The samples were further homogenized with the sonicator and centrifuged at $15,000 \times \mathrm{g}$ for $20 \mathrm{~min}$ at $4^{\circ} \mathrm{C}$. Total protein was quantified using Bio-Rad protein reagent (USA). Crude protein extract, $2 \mathrm{mg}$ each, was transferred to new eppedorf tubes which contained $1 \mathrm{mM} \beta$-NADPH, $2 \mathrm{mM}$ Glucose-6-phosphate, 4 U Glucose-6-phosphate dehydrogenase, $25 \mu \mathrm{M}$ Hemin and solution A. The reactions were performed at $37^{\circ} \mathrm{C}$ in the dark and dried using speed vacuum at $35^{\circ} \mathrm{C}$. The pellet was resuspended with $400 \mu \mathrm{l}$ solution $(50 \% 3 \mathrm{~N} \mathrm{HCl}$ and $50 \%$ 
acetonitrile). The mixtures were centrifuged at $18,350 \times \mathrm{g}$ for $10 \mathrm{~min}$. Supernatants were used to measure the biliverdin concentration with HPLC as described previously. All chemicals used in the heme oxygenase activity assay were purchased from Sigma-Aldrich Inc (USA).

\section{Exogenous biliverdin injection and analysis of eggshell pigment deposition process}

Exogenous biliverdin, $500 \mathrm{nM}$ each, was dissolved in 1X PBS and slowly and gently injected into the shell gland antrum of five laying Pekin ducks at 12-14 $\mathrm{h}$ post oviposition. Eggs from BSD, WSD, WSD with injected biliverdin, as well as $\mathrm{BSH}$ and $\mathrm{BrSH}$, were collected for the eggshell pigmentation deposition process study. The eggs were analyzed using the eggshell deposition method modified from Wang et al. (2007). Eggs were rinsed with deionized water, dried in an oven for $5 \mathrm{~min}$, weighed on a microbalance to obtain the initial weight (No.0) and recorded as W0. Each egg was gently placed into a $150 \mathrm{ml}$ beaker containing $60 \mathrm{ml}$ of solvent (methanol:concentrated $\mathrm{HCl}=2: 1$ ), and incubated for $2 \mathrm{~min}$. The egg was removed from the solvent and rinsed with 3,000 $\mathrm{ml}$ deionized water; then placed back into the oven for 5 min until its surface was dry, weighed again, and recorded as W1. An aliquot of $10 \mathrm{ml}$ solvent in which the No.1 eggshell layer was
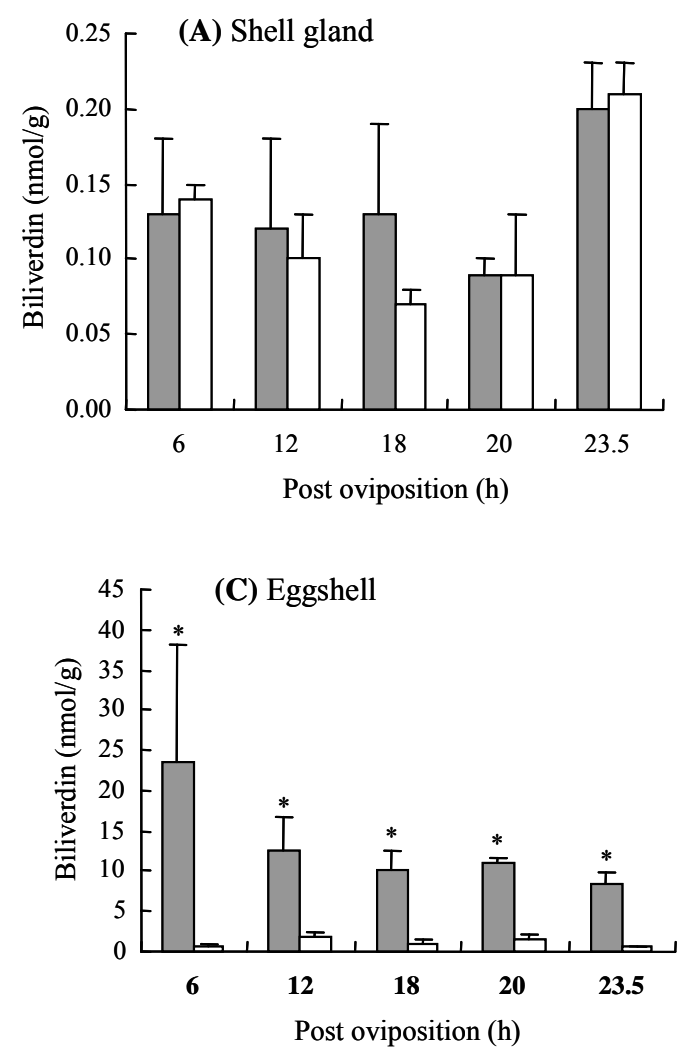

dissolved was transferred into a $15 \mathrm{ml}$ centrifuge tube for analysis. The same egg was placed into another clean beaker and the procedure was repeated until the eggshell was completely dissolved. The $10 \mathrm{ml}$ solution for analysis from each layer was placed in darkness overnight and centrifuged at $10,000 \times \mathrm{g}$ for $10 \mathrm{~min}$. The color of the supernatant solution was analyzed in a spectro-photometer at wavelengths of $412 \mathrm{~nm}$ and $670 \mathrm{~nm}$.

\section{Statistical analysis}

Data for the biliverdin content in the shell gland, uterus fluid and eggshell at various hours post oviposition from BSD and WSD were analyzed using one-way ANOVA. The comparison of means among groups was performed using Tukey's honest significant difference test. All of these analyses were performed with the SAS 9.1.3 package (SAS Institute, 2002).

\section{RESULTS}

The BSD and WSD biliverdin concentrations at 6, 12, 18,20 and $23.5 \mathrm{~h}$ post oviposition in shell gland, uterus fluid, and eggshells are shown in Figure 1. The biliverdin concentration in the BSD shell gland remained constant at 6 , 12 and $18 \mathrm{~h}$, and reached the lowest quantity at $20 \mathrm{~h}$ and

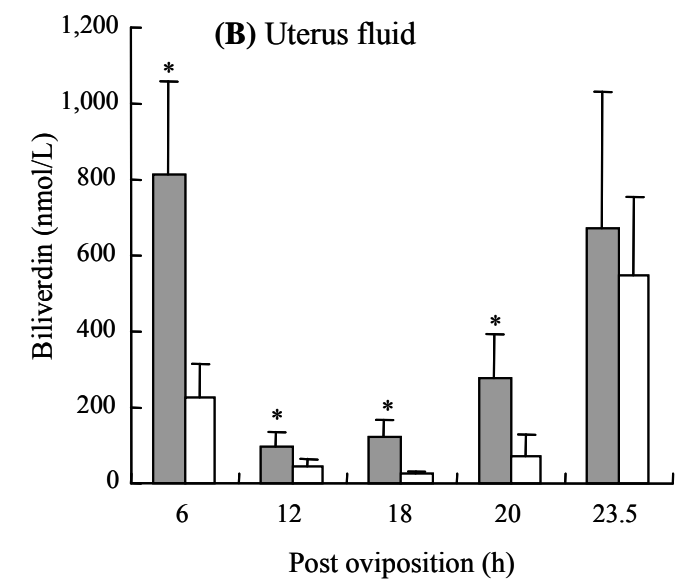

$\square$ Blue-shelled ducks

$\square$ White-shelled ducks

Figure 1. The contents of biliverdin in the shell gland (A), uterus fluid (B) and eggshell (C) at various time points post oviposition for blue-shelled and white-shelled ducks. The bars and whiskers indicate mean \pm SD. $*$ Significantly different between blue-shelled and white-shelled ducks at same hour post oviposition, $\mathrm{p}<0.05$. 
peaked at $23.5 \mathrm{~h}$ post oviposition (Figure 1A). The biliverdin concentration pattern in the shell gland during a WSD ovulatory cycle was similar to that in the blue-shelled counter parts. It gradually decreased from $6 \mathrm{~h}$ and reached the lowest point earlier at $18 \mathrm{~h}$, then peaked at $23.5 \mathrm{~h}$ post oviposition. However, our data was unable to show a significant difference in the comparison for the shell gland biliverdin concentrations in these two breeds.

The biliverdin concentration in the BSD uterus fluid also declined after $6 \mathrm{~h}$ and reached the lowest number at 12 $\mathrm{h}$. It then increased linearly until $23.5 \mathrm{~h}$ post oviposition. The same pattern was observed in the WSD (Figure 1B). The BSD showed higher biliverdin concentration than the WSD and there were significant differences $(p<0.05)$ in the biliverdin concentration in the uterus fluid between these two species, except at $23.5 \mathrm{~h}$. The biliverdin concentration in the eggshell at various hours post oviposition was also measured using spectro-photometery. A significant difference $(p<0.05)$ was observed between the two experimental groups. Trace biliverdin was detected in the WSD eggshell as well (Figure 1C).

The heme oxygenase activity, the synthesizing rate determining enzyme for biliverdin formation, was measured by adding substrates in vitro and its products analyzed using the HPLC method. Its activity in the BSD shell gland was not significantly different from the WSD (Figure 2) and remained mostly constant during the ovulatory cycle.

The WSD eggshell color changed from white to blue after receiving a single dose biliverdin injection (Figure 3 ). The biliverdin and protoporphyrin concentrations in the eggshell layers from BSD, WSD and WSD with exogenous

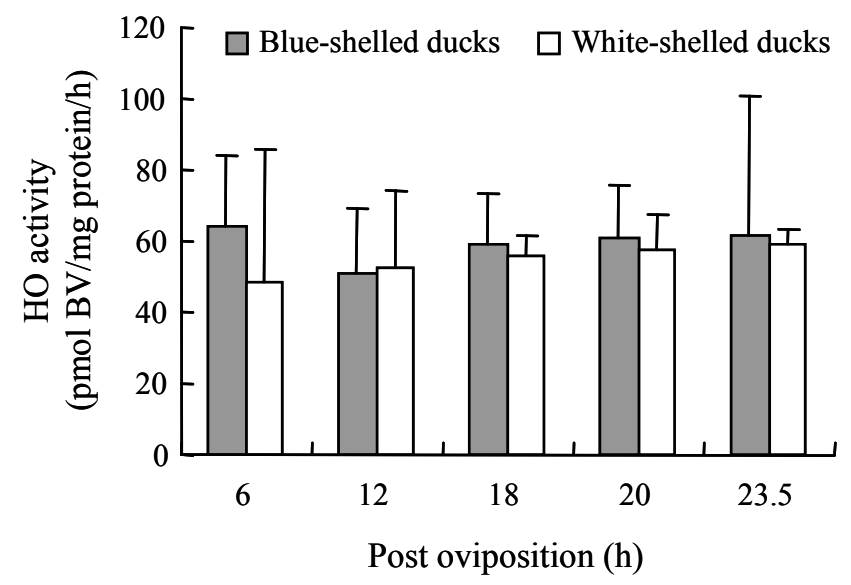

Figure 2. The activity of heme oxygenase in the shell gland at various time points post oviposition for blue-shelled and whiteshelled ducks. The bars and whiskers indicate mean $\pm \mathrm{SD}$.

biliverdin injections are shown in Figure 4. The biliverdin concentrations in the eggshells in the first to sixth BSD layers were significantly higher than those in the WSD, and continued to increase from layer No. 6 to layer No. 1 with a similar trend (Figure 4A). In contrast, the biliverdin concentration in the WSD eggshells was slightly increased from layer No. 5 to layer No. 1. When the exogenous biliverdin was injected into the WSD shell gland antrum, the biliverdin deposition pattern altered. A great quantity of biliverdin appeared in layers 3 and 4, indicating that the exogenous biliverdin was accumulated in this particular eggshell formation period. The protoporphyrin concentration in the WSD eggshells appeared high in the beginning (No. 6 and 7) and the end (No. 1) of eggshell

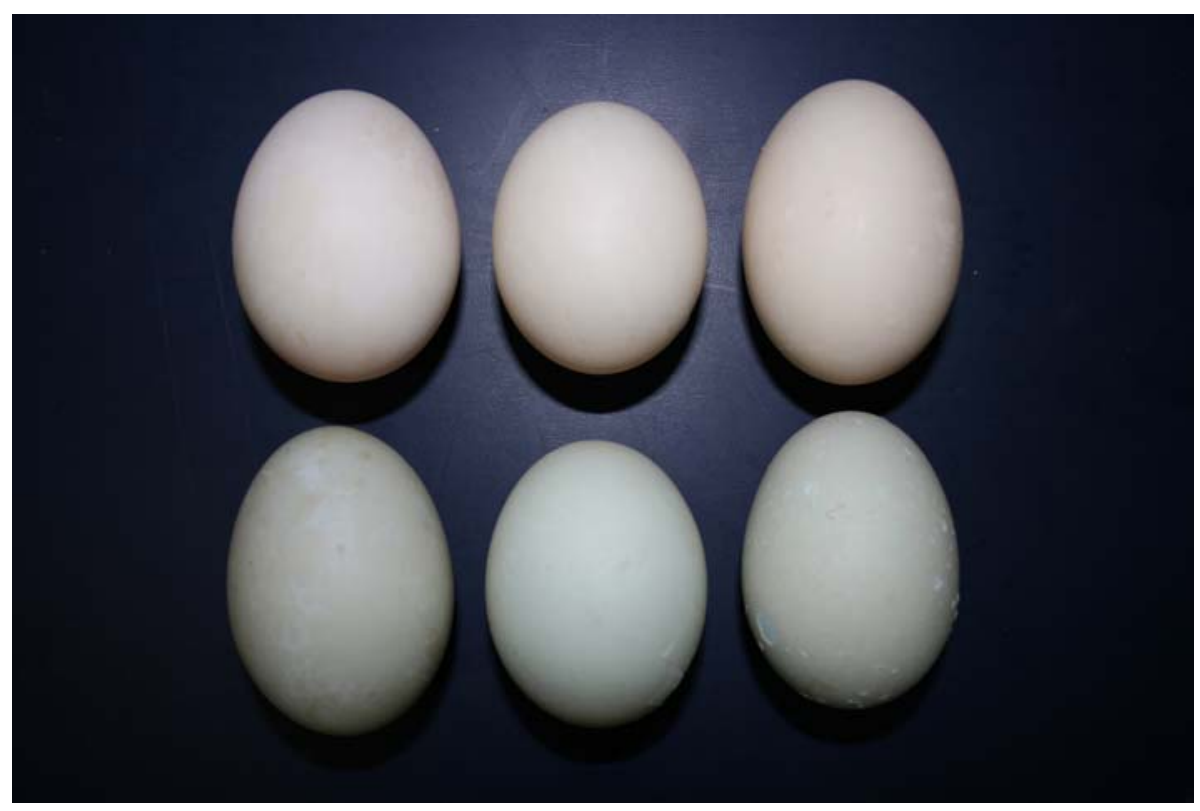

Figure 3. The eggshell color of white-shelled ducks before and after received a single dose biliverdin injection. Upper: the eggs collected before biliverdin injection. Lower: the eggs collected after biliverdin injection. 

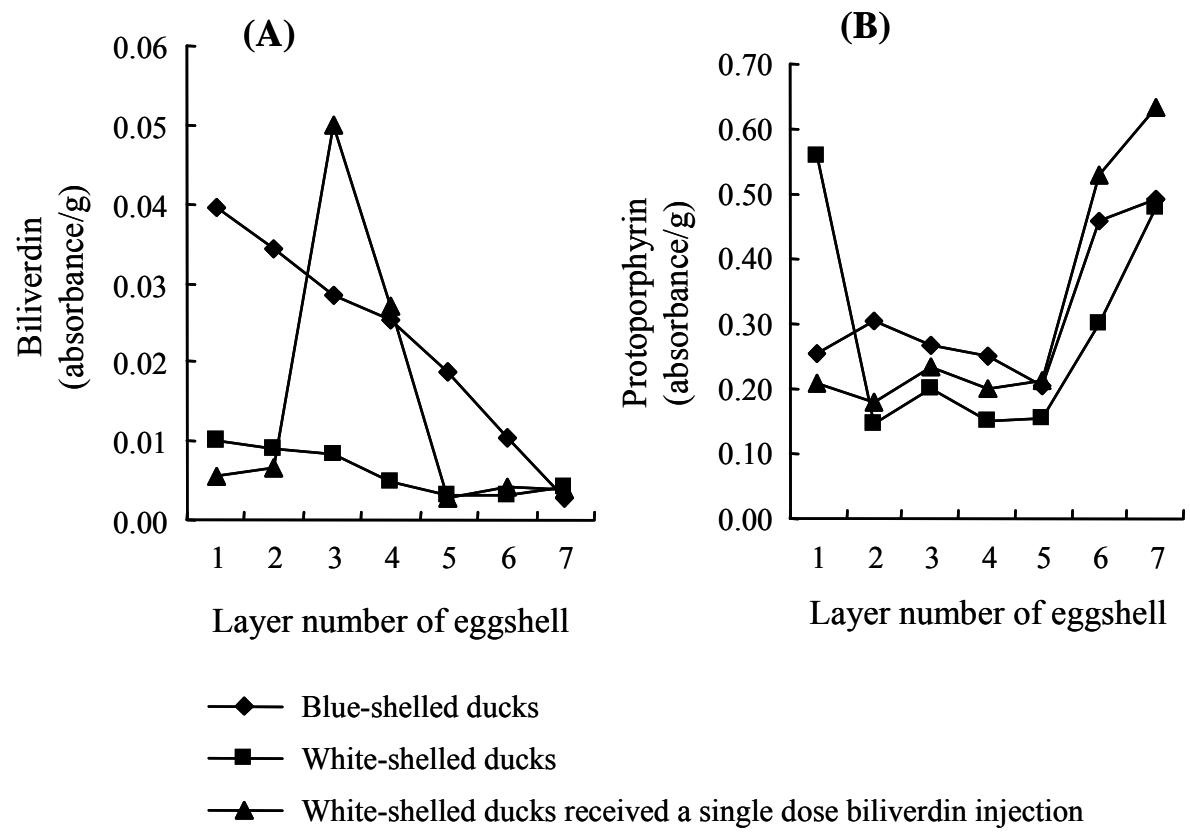

Figure 4. Concentrations of biliverdin (A) and protoporphyrin (B) in the layers of eggshell from blue-shelled ducks, white-shelled ducks and white-shelled ducks received a single dose biliverdin injection.

formation and showed a U-shaped alteration pattern (Figure 4B). The protoporphyrin concentration in the BSD and WSD eggshells that received exogenous biliverdin exhibited the similar pattern as the WSD at layer No. 6 and 7.

\section{DISCUSSION}

The biliverdin deposited in the eggshell was postulated to be derived from either erythrocytes (Kennedy and Vevers, 1973; Lang and Wells, 1987) or from the shell gland (Polin, 1957; Poole, 1966; Baird et al., 1975; Zhao et al., 2006). In a previous report, the biliverdin concentration varied significantly only in the shell gland and not in the circulating serum or bile between $\mathrm{BSH}$ and $\mathrm{BrSH}$. This demonstrates that biliverdin was most likely synthesized in the shell gland and then deposited into the eggshell of chickens (Zhao et al., 2006). In our study there was no significant difference observed in the biliverdin concentration in the shell gland between BSD and WSD. These results may arise from species difference or may implicate different deposition mechanisms for biliverdin for brown-shelled hens and white-shelled ducks. The heme oxygenase, a rate-limited enzyme controls biliverdin production from oxidation and ring opening in the haemoprotein prosthetic groups (Barnett et al., 1975), did not show an activity difference between these two duck species. The static mRNA level of aminolevulenic acid synthase 1 , heme oxygenase 1 and heme oxygenase 2 in the shell gland was also examined in our laboratory. However, there was no significant difference observed (unpublished data). This result is consisted with what Zhao et al. (2007) reported on the genes of related enzymes in the porphyrin pathway applied in the BLAST search and none of these genes were found located inside the blue egg $(O)$ locus. Overall, we were unable to demonstrate any variation for the biliverdin concentration or heme oxygenase activity in the shell gland and the gene expression of relevant enzymes between BSD and WSD.

Eggshell pigments are deposited via ciliated epithelium cells (Johnson, 2000). The shell gland functions as the organ of production, transfer and pigment secretion (Solomom, 2002). Although in trace amount, biliverdin could still be detected in BrSH (Zhao et al., 2006), Coot Fulica atra (Wicke, 1858 as cited in Kennedy and Verers, 1973) and in WSD. Solomon (1991) demonstrated infrared spectra from the brown layer, white layer, quail and blackheaded gull. This evidence does reveal the capacity for both brown and white layers to manufacture and secrete pigment in the shell gland. This study showed significant differences in the biliverdin concentration in the uterus fluid and not in the shell gland between BSD and WSD. These results imply that a mechanism exists that controls the transportation of biliverdin from the shell gland into the uterus fluid and may hold the key to controlling the eggshell color in ducks. Other factors affecting the biliverdin concentration in the uterus fluid should also be considered. This was supported by the eggshell color changing from white to blue when exogenous biliverdin was injected into the WSD shell gland antrum at 12-14 h post oviposition. All of our evidences 
pointed out that it is the biliverdin concentration in the uterus fluid that makes the difference in the eggshell color in ducks.

The egg remains in the shell gland for 18-19 $\mathrm{h}$ in Tsaiya ducks and 18-20 $\mathrm{h}$ in hens (Ma, 1968; Solomon, 1991). The previous study showed, in $\mathrm{BrSH}$ and turkeys, the pigment was deposited during the entire shell formation period with the depository rate accelerated in the last 3 to $5 \mathrm{~h}$ before oviposition (Warren and Conrad, 1942). In addition, the eggshell pigmentation in China domesticated Dongxiang blue-shelled chickens was considered to reach a peak at about 2 to $3 \mathrm{~h}$ before oviposition (Zhao et al., 2006). The eggshell pigment concentration in the top layer was the highest compared with the other layers. These pigments were mainly biliverdin and protoporphyrin in blue eggshells or protoporphyrin in brown eggshells (Wang et al., 2007). Eggs from BSD, WSD, WSD treated with exogenous biliverdin injections were all included in the eggshell pigmentation deposition study. However, BSD eggshell did not contain a large quantity of biliverdin in the top layer (No 1), as shown in the study by Wang et al. (2007). The pigment tended to increase layer by layer. Our result was not consistent with previous reports on the depository patterns of BSH eggshells. It is clear that the eggshell pigmentation deposition pattern indeed differed between the $\mathrm{BSD}$ and BrSH. The biliverdin existed in combination with calcium in the shell cuticle (Kennedy and Verers, 1973; Nakano et al., 2003). The calcium deposition rate was high 10 to $20 \mathrm{~h}$ post oviposition compared with other stages (Chen, 2000). The biliverdin deposition pattern in BSD eggshells was concurrent with the biliverdin concentration in the uterus fluid, which increased from 12 to $23.5 \mathrm{~h}$ post oviposition. With the administration of exogenous biliverdin to the shell gland anstrum, the pigment accumulated continuously onto originally white-shelled eggs until the given biliverdin was exhausted. Our result indicated that the higher the biliverdin concentration in the uterus fluid, the more biliverdin was deposited onto the eggshell. There may be a biliverdin carrier (or biliverdin binding protein) located in the BSD shell gland that enriched biliverdin in the uterus fluid and then deposited into the eggshell. WSD without or functioned less as a carrier (or binding protein) on the shell gland, with almost same biliverdin concentration in the shell gland as BSD. These eggshells remained white. Regardless of eggshell biliverdin being synthesized in the shell gland or transported from the liver or blood, our results indicated that there were no significantly different shell gland biliverdin concentrations between BSD and WSD. The efficiency of the mechanism controlling the transportation of biliverdin from the shell gland into the uterus fluid plays the key role in regulating the eggshell color in ducks. However, the protoporphyrin concentration in the latest layer of such eggs was also different from originally whiteshelled eggs. This may indicate that protoporphyrin deposition was affected directly or indirectly by biliverdin.

Our study demonstrated that the different eggshell colors in BSD and WSD were influenced by the amount of biliverdin in the uterus fluid and not determined by the amount of biliverdin in the shell gland. The eggshell pigment depository pattern in BSD tended to increase layer by layer and was different from that in $\mathrm{BrSH}$, showing a pigment concentration peak in the top eggshell layer.

\section{REFERENCES}

Baird, T., S. E. Solomon and D. R. Tedstone. 1975. Localisation and characterisation of egg shell porphyrin in several avian species. Br. Poult. Sci. 16:201-208.

Barnett, G. M., M. F. Hudson and K. M. Smith. 1975. Concerning of meso-tetraphenylporphyrin purification. J. Chem. Soc. 14:1041-1043.

Chen, D. T., S. R. Lee, Y. H. Hu, C. C. Huang, Y. S. Cheng, C. Tai, J. P. Poivey and R. Rouvier. 2003. Genetic trends for laying traits in the Brown Tsaiya (Anas platyrynchos) selected with restricted genetic selection index. Asian-Aust. J. Anim. Sci. 16(12):1705-1710.

Cornelius, C. E. 1991. Bile pigments in fishes: a review. Vet. Clin. Pathol. 20:106-115.

Johnson, A. L. 2000. Reproduction in the female. In: Sturkie's avian physiology (Ed. G. C. Whittow). Academic press, USA. pp. 569-596.

Gosler, A. G., J. P. Higham and S. J. Reynolds. 2005. Why are birds' eggs speckled? Ecol. Lett. 8(10):1105-1113.

Kennedy, G. Y. and H. G. Ververs. 1973. Eggshell pigments of the Arauano fowl. Comp. Biochem. Physiol. 44B:11-35.

Kennedy, G. Y. and H. G. Ververs. 1975. A survey of avian eggshell pigments. Comp. Biochem. Physiol. 55B:117-123.

Lang, M. R. and J. W. Wells. 1987. A review of eggshell pigmentation. World's Poult. Sci. J. 43(3):238-246.

Liu, S. C., J. F. Huang, T. J. Sun, S. R. Lee and C. T. Wang. 1998. The inheritance of blue eggshell in Brown Tsaiya. J. Taiwan Lives. Res. 31(4):373-382.

Ma, R. C. S. 1968. The time of release of the luteinizing hormone from the adenohypophysis of laying domestic ducks. Poult. Sci. 47:404-410.

Mikšík, I., V. Holáň and Z. Deyl. 1994. Quantification and variability of eggshell pigment content. Comp. Biochem. Physiol. 109A:769-772.

Moreno, J. and J. L. Osorno. 2003. Avian egg colour and sexual selection: does eggshell pigmentation reflect female condition and genetic quality? Ecol. Lett. 6(9):803-806.

Nakano, T., N. I. Ikawa and L. Ozimek. 2003. Chemical composition of chicken eggshell and shell membranes. Poult. Sci. 82:510-514.

Polin, D. 1957. Formation of porphyrin from delta-aminolevulenic acid by uterine and liver tissue from laying hens. Proc. Soc. Exp. Biol. Med. 94:276-279.

Poole, H. K. 1965. Spectrophotometric identification of eggshell pigments and timing of supercial pigment deposition in the Japanese quail. Proc. Soc. Exp. Biol. Med. 119:547-551. 
Ryter, S. W., E. Kvam and R. W. Tyrrell. 2000. Heme oxygenase activity. Methods Mol. Biol. 99:369-391.

SAS Institute Inc. 2002. SAS Qualification Tools User's Guide, version 9.1.3 SAS Institute Inc., Cary, North Carolina.

Schwartz, S. W., A. Raux, B. A. Schacter, B. D. Stephenson and R. N. Shoffner. 1980. Loss of hereditary uterine protoporphyria through chromosomal rearrangement in mutant Rhode Island Red hens. Int. J. Biochem. 12:935-940.

Solomon, S. E. 1991. Eggshell colour. In: Egg and eggshell quality. Wolfe publishing Ltd. England. pp. 123-129.

Solomon, S. E. 2002. The oviduct in chaos. World's Poult. Sci. J. 58:41-48.

Tai, C., R. Rouvier and J. P. Poivey. 1989. Genetic parameters of some growth and egg production traits in laying Brown Tsaiya. Genet. Sel. Evol. 21:377-384.
Wang, X. T., X. M. Deng, C. J. Zhao, J. Y. Li, G. Y. Xu, L. S. Lian and C. X. Wu. 2007. Study of the deposition process of eggshell pigments using an improved dissolution method. Poult. Sci. 86:2236-2238.

Warren, D. C. and R. M. Conrad. 1942. Time of pigment deposition in brown shelled hen eggs and in turkey eggs. Poult. Sci. 21:515-520.

Wicke, W. 1858. Ueber das pigment in den Eischalen der Vögel. Naumannia. 8:393-397.

Zhao, R., G. Y. Xu, Z. Z. Liu, X. Y. Li and N. Yang. 2006. A study on eggshell pigmentation: Biliverdin in blue-shelled chickens. Poult. Sci. 85:546-549.

Zhao, R., Z. Z. Liu, G. Y. Xu and N. Yang. 2007. Analysis of SNP markers for chicken blue-shelled gene using PCR-SSCP. Chin. J. Agric. Biotech. 4(1):53-56. 